\title{
Quark Transversity Distribution in Perturbative QCD: Light-Front Hamiltonian Approach
}

\author{
Asmita Mukherjee* and Dipankar Chakrabarti ${ }^{\dagger}$ \\ Saha Institute of Nuclear Physics \\ 1/AF Bidhannagar, Calcutta 700064, India
}

(12 th February, 2001)

\begin{abstract}
To resolve the current ambiguity in the splitting function corresponding to the quark transversity distribution $h_{1}(x)$, we calculate $h_{1}(x)$ for a dressed quark in light-front Hamiltonian perturbation theory. Our result agrees with the expected form of the splitting function found in the literature and disagrees with the recent calculation in [1]. We emphasize the importance of quark mass in $h_{1}(x)$ in perturbative QCD and show its connection with a part of $g_{T}$.
\end{abstract}

PACS: 11.10.Ef, 12.38.-t, 13.60.Hb

Keywords: transversity distribution, light-front Hamiltonian perturbation theory, splitting function

\section{Introduction}

The quark transversity distribution $h_{1}(x)$ has received some attention lately as a recent calculation [1] of its corresponding splitting function does not agree with the expected result found in the literature [2]. The transversity distribution function does not appear in polarized inclusive deep inelastic scattering in the limit of massless quark, because it corresponds to a flip of quark chirality which is absent in DIS when quark mass is zero. $h_{1}(x)$ can be measured in polarized Drell-Yan process. $h_{1}(x)$ was first discussed by Ralston and Soper for this process [3]. Since then, there has been a lot of theoretical investigations and also several attempts to experimentally measure the transversity distribution (see [1, [1] and references therein). However, the effect of quark mass has not been discussed so far.

The leading contribution of the transversity distribution is twist two and it gives the difference of quark densities with eigenvalues $\frac{1}{2}$ and $-\frac{1}{2}$ in the eigenstates of the transverse Pauli-Lubanski spin operator. In order to calculate its corresponding splitting function,

*email: asmita@theory.saha.ernet.in

†email: dipankar@theory.saha.ernet.in 
Artru and Mekhfi analyzed the helicity amplitude of hadron-hadron scattering in the $t$ channel and used Altarelli-Parisi evolution equation. The splitting function calculated by them is given by in the limit of zero quark mass,

$$
P_{s_{\perp}}(x)=\frac{C_{f}}{2}\left[\frac{4}{(1-x)_{+}}-4+3 \delta(1-x)\right],
$$

where the color factor $C_{f}=\frac{N^{2}-1}{2 N}$ for $S U(N)$. The delta function comes from a counterterm which is the same as in the helicity non-flip splitting function, $P_{q q}(z)$.

However in a recent work, Hermann, Kuhn and Schutzhold have calculated the splitting function for $h_{1}$ and their result does not agree with Artru et al [1]. They have started with the forward scattering amplitude $T_{\mu}$ which contribute to the nonsinglet transversity distribution. By Wick expansion of the products of currents in the amplitude, they found that 32 Feynman diagrams contribute to the splitting function, although considering various constraints on $T$, they showed that calculation of 8 Feynman diagrams is sufficient for their purpose. They have taken the quark mass to be zero. The singularity structure of the splitting function is found by using a dispersion relation and the splitting functions are obtained by doing inverse Mellin transformation. The splitting function is given by,

$$
P_{\perp}(x)=\frac{C_{f}}{(4 \pi)^{2}}\left(4-\frac{4}{(1-x)_{+}}\right)
$$

and the corresponding anomalous dimension

$$
\gamma_{n}=\frac{C_{f}}{(4 \pi)^{2}}\left(\frac{4}{1+n}+4 S_{n}\right),
$$

where

$$
S_{n}=\sum_{j=1}^{n} \frac{1}{j} .
$$

The splitting function differs from the earlier result by a delta function. They have also calculated $g_{1}(x)$ in the same method. $g_{1}(x)$ calculated in this method agrees with the expected result in the literature. On comparing the two calculations, they have shown that the splitting function for $g_{1}$ contains a delta function that comes from inverse Mellin transformation. The behavior at $x=1$ is usually fixed by assuming a sum rule, whereas no such sum rule is there for $h_{1}(x)$. They pointed out that in the earlier works, the behavior of $h_{1}$ at $x=1$ has been fixed by hand.

To resolve the above confusion generated by mutually contradictory results, we calculate the transversity distribution in an entirely different method based on light-front Hamiltonian technique. To uncover the physical picture of $h_{1}(x)$, it is most useful to go to light-front formalism where the polarized and unpolarized parton distributions in hard scattering can be expressed as the light-cone Fourier transform of the matrix element of bilocal operators in light-front gauge $A^{+}=0$ [0]. In this method we can directly calculate the structure function itself and hence we do not need to do any inverse Mellin transformation as other formalisms need. Also, the role played by the quark mass can be seen clearly in this picture. We then compare $h_{1}(x)$ with the transverse polarized structure function $g_{T}$. 


\section{Transversity Distribution $h_{1}(x)$ in Light-Front Hamiltonian Perturbation The- ory}

The leading contribution to the transversity distribution function $h_{1}(x)$ in $A^{+}=0$ gauge is given by [5]

$$
h_{1}(x)=\frac{1}{P^{+}} \int \frac{d \lambda}{2 \pi} e^{i \lambda x}\left\langle P S_{\perp}\left|\psi_{+}^{\dagger}(0) \gamma_{\perp} \gamma_{5} \psi_{+}\left(y^{-}\right)\right| P S_{\perp}\right\rangle
$$

Here $\lambda=\frac{1}{2} P^{+} y^{-}$and $\psi=\psi^{+}+\psi^{-}$, where $\psi^{ \pm}=\Lambda^{ \pm} \psi$, the projection operators $\Lambda^{ \pm}=\frac{1}{4} \gamma^{ \pm} \gamma^{\mp}$. The above expression differs from Jaffe and Ji by a factor of $\sqrt{2}$ in the denominator because of our different convention. $P^{\mu}$ and $S^{\mu}$ are the total momentum and polarization of the target state. The operator in $h_{1}(x)$ does not involve the constrained field $\psi^{-}$, so is a 'good' operator. Because of this, unlike the transverse structure function $g_{T}, h_{1}$ has a simple parton interpretation. Also, here we are calculating $h_{1}(x)$ which is a gauge invariant object, in the light-front gauge $A^{+}=0$. In this gauge, the link operator involved in the bilocal expression is unity. For simplicity, we have not considered the flavor dependence.

The representation of the light-front $\gamma$-matrix we use here is the following

$$
\gamma^{0}=\left[\begin{array}{cc}
0 & -i \\
i & 0
\end{array}\right], \quad \gamma^{3}=\left[\begin{array}{cc}
0 & i \\
i & 0
\end{array}\right], \quad \gamma^{i}=\left[\begin{array}{ccc}
-i \tilde{\sigma}^{i} & 0 \\
0 & i \tilde{\sigma}^{i}
\end{array}\right]
$$

where $\tilde{\sigma}^{1}=\sigma^{2}, \quad \tilde{\sigma}^{2}=-\sigma^{1}$. The expression Eq.(4) in general is nonperturbative and the target is a bound state. However, this expression can be used in pertubative calculation of the splitting function corresponding to $h_{1}(x)$ in a straightforward manner by replacing the target state by a dressed quark state in light-front Hamiltonian perturbation theory.

The transversely polarized state can be expressed in terms of helicity states as,

$$
\left|P S_{\perp}\right\rangle=\frac{1}{\sqrt{2}}(|P \uparrow\rangle \pm|P \downarrow\rangle)
$$

Without any loss of generality, we take the state to be polarized along $x$ direction. The polarization vector $S^{\mu}$ is defined as,

$$
S^{\mu}=\frac{1}{2} \bar{u} \gamma^{\mu} \gamma^{5} u
$$

where $u$ is the Dirac spinor. The components of the above polarization vector can be calculated for both longitudinal and transverse polarization. For longitudinal polarization, we get, $S^{+}=P^{+}, S^{\perp}=0$. However for transverse polarization along $x$ direction, the components are, $S^{2}=0, S^{1}=M, S^{-}=2 \frac{P^{1}}{P^{+}} M, S^{+}=0$ where $M$ is the mass of the state. It is clear that one needs a massive state in order to have transverse polarization.

A dressed quark state of total momentum $P$ and helicity $\sigma$ can be expanded in Fock space as,

$$
\begin{aligned}
|P \sigma\rangle=\sqrt{N} & \left\{b_{\sigma}^{\dagger}(P)|0\rangle+\sum_{\sigma_{2}, \lambda_{2}} \int \frac{d k_{1}^{+} d^{2} k_{1}^{\perp}}{\sqrt{2(2 \pi)^{3} k_{1}^{+}}} \int \frac{d k_{2}^{+} d^{2} k_{2}^{\perp}}{\sqrt{2(2 \pi)^{3} k_{2}^{+}}} \psi_{2}\left(P, \sigma \mid k_{1}, \sigma_{2} ; k_{2}, \lambda_{2}\right)\right. \\
& \left.\times \sqrt{2(2 \pi)^{3} P^{+}} \delta^{3}\left(P-k_{1}-k_{2}\right) b_{\sigma_{2}}^{\dagger}\left(k_{1}\right) a_{\lambda_{2}}^{\dagger}\left(k_{2}\right)|0\rangle+\ldots\right\}
\end{aligned}
$$


where $N$ is the normalization constant and $\psi_{2}\left(P, \sigma \mid k_{1}, \sigma_{2} ; k_{2}, \lambda_{2}\right)$ is the probability amplitude to find a bare quark with momentum $k_{1}$ and helicity $\sigma_{2}$ and a bare gluon with momentum $k_{2}$ and helicity $\lambda_{2}$ in the dressed quark. Here, we consider upto two-particle sector in the Fock-space.

We introduce the Jacobi momenta

$$
x_{i}=\frac{k_{i}^{+}}{P^{+}}, \quad \kappa_{i}^{\perp}=k_{i}^{\perp}-x_{i} P^{\perp}
$$

so that

$$
\sum_{i} x_{i}=1, \quad \sum_{i} \kappa_{i}^{\perp}=0 .
$$

We redefine $\psi_{2}$ in a boost invariant form

$$
\sqrt{P^{+}} \psi_{2}\left(k_{i}^{+}, k_{i}^{\perp}\right)=\Phi_{2}\left(x_{i}, \kappa_{i}^{\perp}\right) .
$$

The two particle amplitude is given by [6]

$$
\begin{aligned}
\Phi_{2}^{\sigma_{2}, \lambda_{2}}\left(x, \kappa^{\perp} ; 1-x,-\kappa^{\perp}\right)= & \frac{1}{\left[m^{2}-\frac{m^{2}+\kappa^{\perp^{2}}}{x}-\frac{\kappa^{\perp^{2}}}{1-x}\right]} \frac{1}{\sqrt{1-x}} T^{a} \chi_{\sigma_{2}}^{\dagger} \\
& \times\left[-2 \frac{\kappa^{\perp}}{1-x}-\frac{\sigma^{\perp} \cdot \kappa^{\perp}-i m}{x} \sigma^{\perp}-\sigma^{\perp} i m\right] \chi_{\sigma} \cdot\left(\epsilon_{\lambda_{2}}^{\perp}\right)^{\star} .
\end{aligned}
$$

Here $m$ is the mass of the quark and $\chi_{\sigma}$ is the two component spinor for the quark and $\epsilon_{\lambda_{2}}^{\perp}$ is the gluon polarization vector.

We first calculate the matrix element contribution to $h_{1}(x)$ between two helicity states (of helicity $\sigma$ and $\sigma^{\prime}$ ) of dressed quarks,

$$
h^{\prime}(x)=\frac{1}{P^{+}} \int \frac{d \lambda}{2 \pi} e^{i \lambda x}\left\langle P \sigma\left|\psi_{+}^{\dagger}(0) \gamma_{\perp} \gamma_{5} \psi_{+}\left(y^{-}\right)\right| P \sigma^{\prime}\right\rangle .
$$

Since the state is polarized along $x$ direction, $\gamma_{\perp}=\gamma_{1}$. In the two component formalism

$$
\psi_{+}(x)=\left[\begin{array}{c}
\xi(x) \\
0
\end{array}\right]
$$

and the chirality flip operator reduces to

$$
\psi_{+}^{\dagger}(0) \gamma_{\perp} \gamma_{5} \psi_{+}(y)=-\xi^{\dagger}(0) \sigma^{1} \xi(y)
$$

with

$$
\xi(x)=\sum_{\lambda} \chi_{\lambda} \int \frac{d k^{+} d^{2} k^{\perp}}{2(2 \pi)^{3} \sqrt{k^{+}}}\left[b_{\lambda}(k) e^{-i k x}+d_{-\lambda}^{\dagger}(k) e^{i k x}\right] .
$$

At this stage, the mass terms in the vertex Eq.(9) contains $\sigma^{\perp}$ which flips helicity. These terms give zero contribution in $h_{1}$ because of the $\sigma^{1}$ present in the operator. We now have 


$$
\begin{aligned}
h^{\prime}(x) & =\frac{1}{P^{+}} \int \frac{d \lambda}{2 \pi} e^{i \lambda x}\left\langle P \sigma\left|-\xi^{\dagger}(0) \sigma^{1} \xi(y)\right| P \sigma^{\prime}\right\rangle \\
& =-N\left[\delta(1-x)+\frac{\alpha_{s} C_{f}}{4 \pi^{2}} \frac{4 x}{1-x} \int d^{2} \kappa^{\perp} \frac{1}{\left(\kappa^{\perp}\right)^{2}}\right]\left(\chi_{\sigma^{\dagger}}^{\dagger} \chi_{\sigma^{\prime}}\right) .
\end{aligned}
$$

The first term comes from the single particle sector and the second term comes from the two-particle (one quark and one gluon) sector. Regulating the lower limit by $\mu$ and upper limit by $\mathrm{Q}$ in $\kappa$ integration we get

$$
h^{\prime}(x)=-N\left[\delta(1-x)+\frac{\alpha_{s} C_{f}}{2 \pi} \frac{2 x}{1-x} \ln \frac{Q^{2}}{\mu^{2}}\right]\left(\chi_{\sigma}^{\dagger} \sigma^{1} \chi_{\sigma^{\prime}}\right) .
$$

$h^{\prime}(x) \neq 0$ only if $\sigma \neq \sigma^{\prime}$.

The normalization of the state

$$
\left\langle P \sigma \mid P^{\prime} \sigma^{\prime}\right\rangle=2(2 \pi)^{3} P^{+} \delta\left(P^{+}-P^{\prime+}\right) \delta^{2}\left(P^{\perp}-P^{\perp}\right) \delta_{\sigma, \sigma^{\prime}}
$$

gives,

$$
N\left[1+\frac{\alpha_{s}}{2 \pi} C_{f} \int_{\epsilon}^{1-\epsilon} d x \frac{1+x^{2}}{1-x} \ln \frac{Q^{2}}{\mu^{2}}\right]=1 .
$$

Upto order $\alpha_{s}$ we get [6]

$$
N=1-\frac{\alpha_{s}}{2 \pi} C_{f} \int_{\epsilon}^{1-\epsilon} d x \frac{1+x^{2}}{1-x} \ln \frac{Q^{2}}{\mu^{2}} .
$$

It is important to mention here that, the mass terms in the vertex in Eq.(9) gives $m^{2}$ terms in the normalization condition, which are power suppressed. We have neglected such contributions. In the denominator, $m^{2}$ is neglected compared to $\left(\kappa^{\perp}\right)^{2}$ since $m^{2}<<\left(\kappa^{\perp}\right)^{2}$. Here $\epsilon$ is a small cutoff on the longitudinal momentum fraction $x$ which can be safely taken to be zero at the end of the calculation. Using the definition Eq.(6) for the transversely polarized state in terms of the helicity states, we get the transversity distribution function

$$
h_{1}(x)=\delta(1-x)+\frac{\alpha_{s}}{2 \pi} C_{f} \ln \frac{Q^{2}}{\mu^{2}}\left[\frac{2 x}{1-x}-\delta(1-x) \int_{\epsilon}^{1-\epsilon} d y \frac{1+y^{2}}{1-y}\right]
$$

which can be written as,

$$
h_{1}(x)=\delta(1-x)+\frac{\alpha_{s} C_{f}}{2 \pi}\left(\frac{2}{(1-x)_{+}}-2+\frac{3}{2} \delta(1-x)\right) \ln \frac{Q^{2}}{\mu^{2}} .
$$

The splitting function can be easily obtained from the above expression:

$$
P_{s_{\perp}}(x)=\frac{C_{f}}{2}\left[\frac{4}{(1-x)_{+}}-4+3 \delta(1-x)\right]
$$

which exactly agrees with Eq.(11). Depending on the plus or minus sign in the definition of $\left|P S_{\perp}\right\rangle$ given by Eq.(6), the result can have an overall minus sign. The anomalous dimension is defined as, 


$$
\gamma_{n}=\int_{0}^{1} d z z^{n-1} P_{s_{\perp}}(z)
$$

Using the above splitting function, we get,

$$
\gamma_{n}=C_{f}\left(\frac{3}{2}-2 \sum_{j=1}^{n} \frac{1}{j}\right)
$$

which again agrees with the expected result found in the literature [2:7]. However, the above analysis is done is a completely different approach based on light-front Hamiltonian perturbation theory. Instead of Feynman diagrams, here we have old-fashioned $x^{+}$ordered diagrams. The complexities of the operator product expansion are absent. There is no need to calculate the moments of the structure functions and then invert it. In this formalism, we get the expressions of the structure functions themselves directly in terms of bilocal matrix elements. As we have shown above, the splitting functions can be obtained easily by calculating the matrix element for a dressed parton state. In our calculation, we have not assumed any sum rule for $h_{1}(x)$. The final result for the splitting function is obtained only after taking the normalization of the state into account.

Another very important point is that, the role played by the quark mass in the perturbative analysis of $h_{1}(x)$ can be seen very clearly in this picture, whereas it is obscure in other formalisms. In order to show clearly the effect of quark mass, we compare our calculation with the calculation of $g_{T}$ in this method.

\section{Comparison with the Transverse Polarized Structure Function $g_{T}$}

It is very interesting to compare and contrast the above calculation with the calculation of $g_{T}$ for a dressed quark in order to understand the effect of quark mass in the two cases. Recently, $g_{T}$ has been calculated in light-front Hamiltonian perturbation theory [8]. $g_{T}$ is given by,

$$
g_{T}\left(x, Q^{2}\right)=\frac{1}{8 \pi\left(S_{\perp}-\frac{P_{\perp}}{P^{+}} S^{+}\right)} \int d \eta e^{-i \eta x}\left\langle P S\left|\left(O_{m}+O_{k_{\perp}}+O_{g}\right)+h . c .\right| P S\right\rangle
$$

where

$$
\begin{aligned}
O_{m} & =m \psi_{+}^{\dagger}\left(\xi^{-}\right) \gamma_{\perp} \frac{1}{i \partial^{+}} \gamma_{5} \psi_{+}(0) \\
O_{k_{\perp}} & =-\psi_{+}^{\dagger}\left(\xi^{-}\right)\left(\gamma_{\perp} \frac{1}{\partial^{-}} \gamma^{\perp} \cdot \partial^{\perp}+2 \frac{P_{\perp}}{P^{+}}\right) \gamma_{5} \psi_{+}(0) \\
O_{g} & =g \psi_{+}^{\dagger}\left(\xi^{-}\right)\left(\gamma^{\perp} \cdot A^{\perp}\left(\xi^{-}\right)\right) \frac{1}{i \partial^{+}} \gamma_{\perp} \gamma_{5} \psi_{+}(0)
\end{aligned}
$$

and h.c. stands for hermitian conjugate. In the above expression, we have used the equation for the constrained field $\psi^{-}$. The operator $O_{m}$ involves $\gamma^{\perp} \gamma^{5}$ which is similar to $h_{1}$. In the dressed quark calculation, the mass terms in the expression of $\Phi_{2}$ do not contribute in $O_{m}$, exactly like $h_{1}$. However, the other parts of the operator involve $\gamma^{\perp} \gamma^{\perp} \gamma^{5}$ which gets nonzero contribution only from the helicity flip (mass dependent) part of the vertex. So in the case of $g_{T}$, the mass of the quark plays a very important role both in the transversely polarized 
state and in the matrix element but in $h_{1}$, though the mass terms in the vertex do not contribute in the matrix element, quark mass is still important in perturbative calculations because one cannot get a transversely polarized dressed quark state in the massless theory. We emphasize that this effect can be seen more clearly in our approach, because it is a Hamiltonian perturbation theory approach and the state plays a very important role.

The contribution of the mass dependent part $O_{m}$ to $g_{T}$ for a dressed quark is given by [8]:

$$
g_{T}^{m}=\frac{1}{2}\left[\delta(1-x)+\frac{\alpha_{s}}{2 \pi} C_{f} \ln \frac{Q^{2}}{\mu^{2}}\left(\frac{2}{1-x}-\delta(1-x) \int_{\epsilon}^{1-\epsilon} d y \frac{1+y^{2}}{1-y}\right)\right] .
$$

The above expression is similar to $h_{1}$ Eq.(19), the additional $x$ in the numerator of the second term in Eq.(19) comes due to the absence of the $\frac{1}{\partial+}$ in the operator. Thus in perturbation theory, we directly see the connection of $h_{1}(x)$ with the mass dependent part of $g_{T}$.

\section{Conclusion}

We have calculated the splitting function corresponding to the transversity distribution upto $O\left(\alpha_{s}\right)$ in a recently developed light-front Hamiltonian QCD approach. The structure function in hard scattering can be directly expressed in terms of the matrix elements of light-front bilocal currents. There is no need to do an inverse Mellin transformation and the splitting functions can be easily extracted. Another advantage over the Altarelli-Parisi method is that here one deals with the probability amplitudes instead of probability densities and real and virtual processes are calculated to the same order without any difficulty. Here we have not assumed any particular behavior of $h_{1}(x)$ at $x=1$. The delta function with the correct coefficient in the final expression comes from the normalization constant of the state.

We have taken the quark mass to be nonzero in our calculation and shown that $h_{1}(x)$ can be related to one part of the transverse polarized structure function $g_{T}$. We have emphasized the importance of quark mass in the perturbative analysis of $h_{1}(x)$. To our knowledge, this is done for the first time in the literature. We emphasize that in light-front Hamiltonian perturbation theory, this effect is seen in a more clear way since it is not a Feynman diagram based calculation.

Our result not only verifies the earlier result of Artru et. al. [2] but does so in a completely different and much more straightforward way. The disagreement with [1] introduces questions regarding the validity of various assumptions made in their paper.

We acknowledge Prof. A. Harindranath for suggesting this problem and also for many useful discussions. 


\section{REFERENCES}

[1] M. M. Hermann, R. Kuhn and R. Schutzhold, hep-ph/0012226.

[2] X. Artru and M. Mekhfi, Z. Phys, C45 (1990) 669.

[3] J. P. Ralston and D. E. Soper, Nucl. Phys. B152 (1979) 109.

[4] B. L. Ioffe and A. Khodjamirian, Phys. Rev. D51 (1995) 3373; E. D. Salvo, hepph/0101277; V. A. Korotkov, W. D. Nowak, hep-ph/0102015; P. Schweitzer, D. Urbano, M. V. Polyakov, C. Weiss, P. V. Pobylitsa, K. Goeke, hep-ph/0101300.

[5] R. L. Jaffe and X. Ji, Phys. Rev. Lett 67 (1991) 552; Nucl. Phys. B375 (1992) 527.

[6] A. Harindranath, R. Kundu and W-M. Zhang, Phys. Rev. D59 (1999) 094012, 094013.

[7] V. Barone, Phys. Lett B409 (1997) 499.

[8] A. Harindranath and W. M. Zhang, Phys. Lett. B408 (1997) 347; A. Harindranath, A. Mukherjee and R. Ratabole, Phys. Lett. B476 (2000) 471; Phys. Rev. D63 (2001) 045006 . 